SECTION 2. Applied mathematics. Mathematical modeling.

Zhunisbekov Sagat doctor of technical Sciences, academician of the National Engineering Academy rector of Taraz Technical Institute, Kazakhstan

Shevtsov Alexandr Nikolayevich candidate of technical Sciences,

President of International Academy of Theoretical \& Applied Sciences, Kazakhstan

\title{
ABOUT ONE MODEL OF THE PROCESS OF CRYSTALLIZATION
}

Abstract: In this paper describes a dynamic mathematical model of the process of crystallization of metal in three-dimensional space.

Key words: model, metal, Delphi, crystallization.

\section{ОБ ОДНОЙ МОДЕЛИ ПРОЦЕССА КРИСТАЛЛИЗАЦИИ}

\author{
Аннотация: В данной статье разрабатывается динамическая \\ математическая модель процесса кристаллизаџии металла в трехмерном \\ пространстве.
}

Ключевые слова: модель, металл, дельфи, кристаллизация.

Расположение атомов в кристалле - кристаллическая решетка - может принимать множество геометрических форм [1]. Рассмотрим сначала простейшие решетки, характерные для большинства металлов и инертных газов в твердом состоянии. Это кубические решетки, которые могут быть двух видов: объемноцентрированная кубическая (Рис.1, а) и гранецентрированная кубическая (Рис.2, б). Здесь, на рисунках показан только один «куб» решетки. Для простоты на рисунке показаны только «центры» атомов. В настоящих кристаллах атомы скорее похожи на соприкасающиеся друг с другом шарики. Темные и светлые шарики на приведенных рисунках могут, вообще говоря, означать либо разные, либо одинаковые сорта атомов. Так, железо имеет объемноцентрированную кубическую решетку при низких температурах и гранецентрированную кубическую решетку при более высоких температурах. Физические свойства этих двух кристаллических форм совершенно различны.
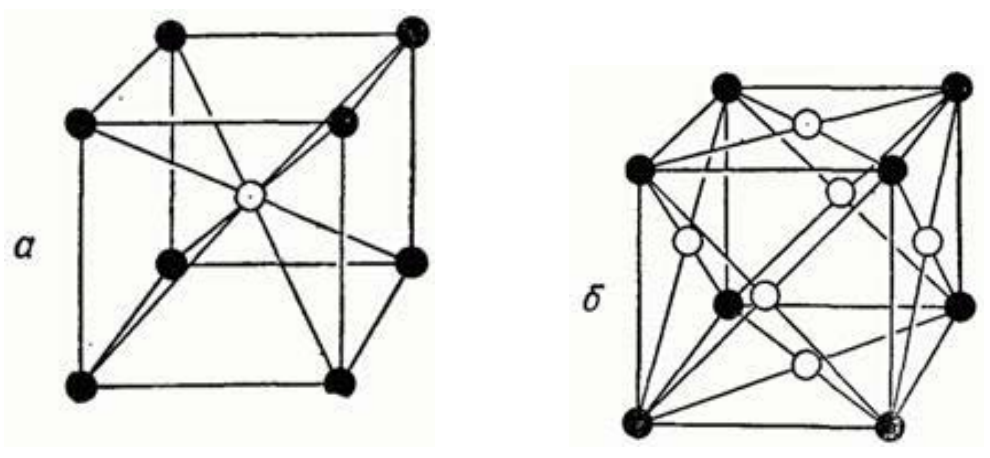

Рисунок 1 - Элементарная ячейка кубического кристалла.

(а - объемноцентрированная; б - гранецентрированная) 
Рассмотрим процесс образования подобных ячеек кристалла. Мы должны как можно плотнее упаковать атомы - шарики. Обозначим сторону куба через $a$, a координаты серединного атома через $(x, y, z)$, тогда координаты остальных атомов можно будет определить по формулам:

$$
\begin{array}{ll}
\left(x+\frac{a}{2}, y+\frac{a}{2}, z+\frac{a}{2}\right) & \left(x+\frac{a}{2}, y+\frac{a}{2}, z-\frac{a}{2}\right) \\
\left(x+\frac{a}{2}, y-\frac{a}{2}, z+\frac{a}{2}\right) & \left(x+\frac{a}{2}, y-\frac{a}{2}, z-\frac{a}{2}\right) \\
\left(x-\frac{a}{2}, y+\frac{a}{2}, z+\frac{a}{2}\right) & \left(x-\frac{a}{2}, y+\frac{a}{2}, z-\frac{a}{2}\right) \\
\left(x-\frac{a}{2}, y-\frac{a}{2}, z+\frac{a}{2}\right) & \left(x-\frac{a}{2}, y-\frac{a}{2}, z-\frac{a}{2}\right)
\end{array}
$$

Для определенности будем рассматривать атомы как центральное ядро диаметром 0,1анг. и диаметр самого атома порядка 1 анг. Поместим в объем 10 анг. ${ }^{3}$ не более 1000 атомов. Зададим для каждого из них пространственную координату, единичный вектор скорости и уровень кинетической энергии в пределах [0...1]. Причем при 30\% энергии, и менее, атом будет прекращать свое движение.

Процесс кристаллизации возможен только из жидкого состояния, а значит для всех атомов будут выполняться законы столкновения частиц характерные для жидких или газообразных сред. Внешней гравитацией на первом этапе будем пренебрегать.

Уровень энергии в начальный момент для всех атомов будет максимальным и может характеризоваться как - температура нагрева металла. Также будем отображать оси координатной системы, и элемент размерности (Рис.2).

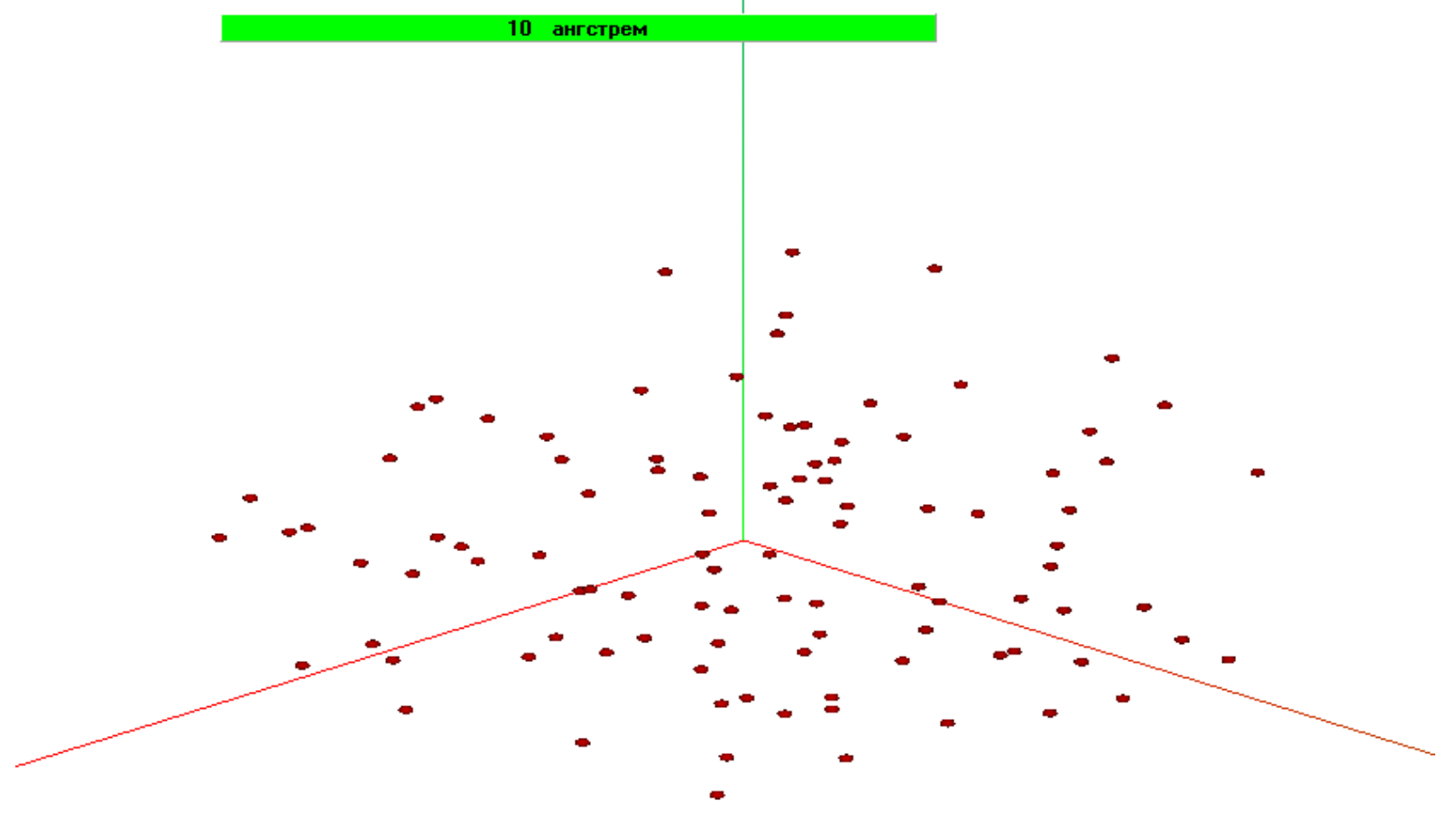

Рисунок 2 - Модель кристаллизации, 100 атомов. 
Полученный процесс перемещения атомов удобно наблюдать - вращая систему координат вокруг оси Oz, (Рис.3).

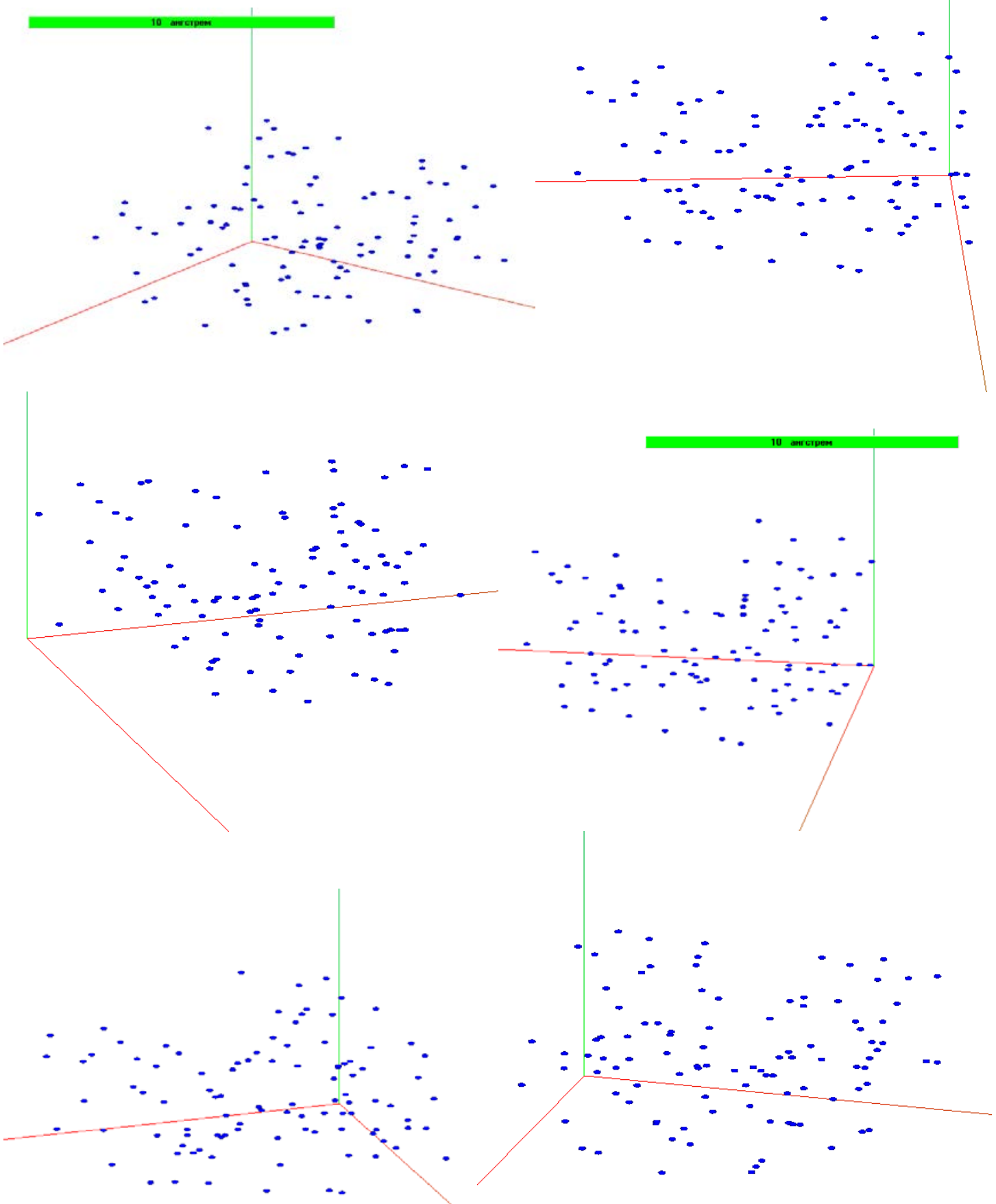

Рисунок 3 - Вращение модели в пространстве вокруг Oz.

Цвет атома характеризует степень его нагрева и меняется динамически от красного к синему, при охлаждении. Атомы находятся в непрерывном движении и при соударении общая энергия определяется как полусумма энергий атомов до соударения. Также происходит обмен векторами направления движения. 
Увеличим число атомов до 1000, (Рис.4). Зададим граничные условия на оддной из граней следующим образом:

$$
\left.E_{\text {кин }}(\text { i })\right|_{i=1 \ldots 1000}=90 \% * E_{\text {кин }}(i)
$$

Что характеризует - охлаждение куба и отток $10 \%$ тепловой энергии через грань, при каждом соударении. Процесс охлаждения на 70\% занимает всего 39 секунд (до полной остановки).

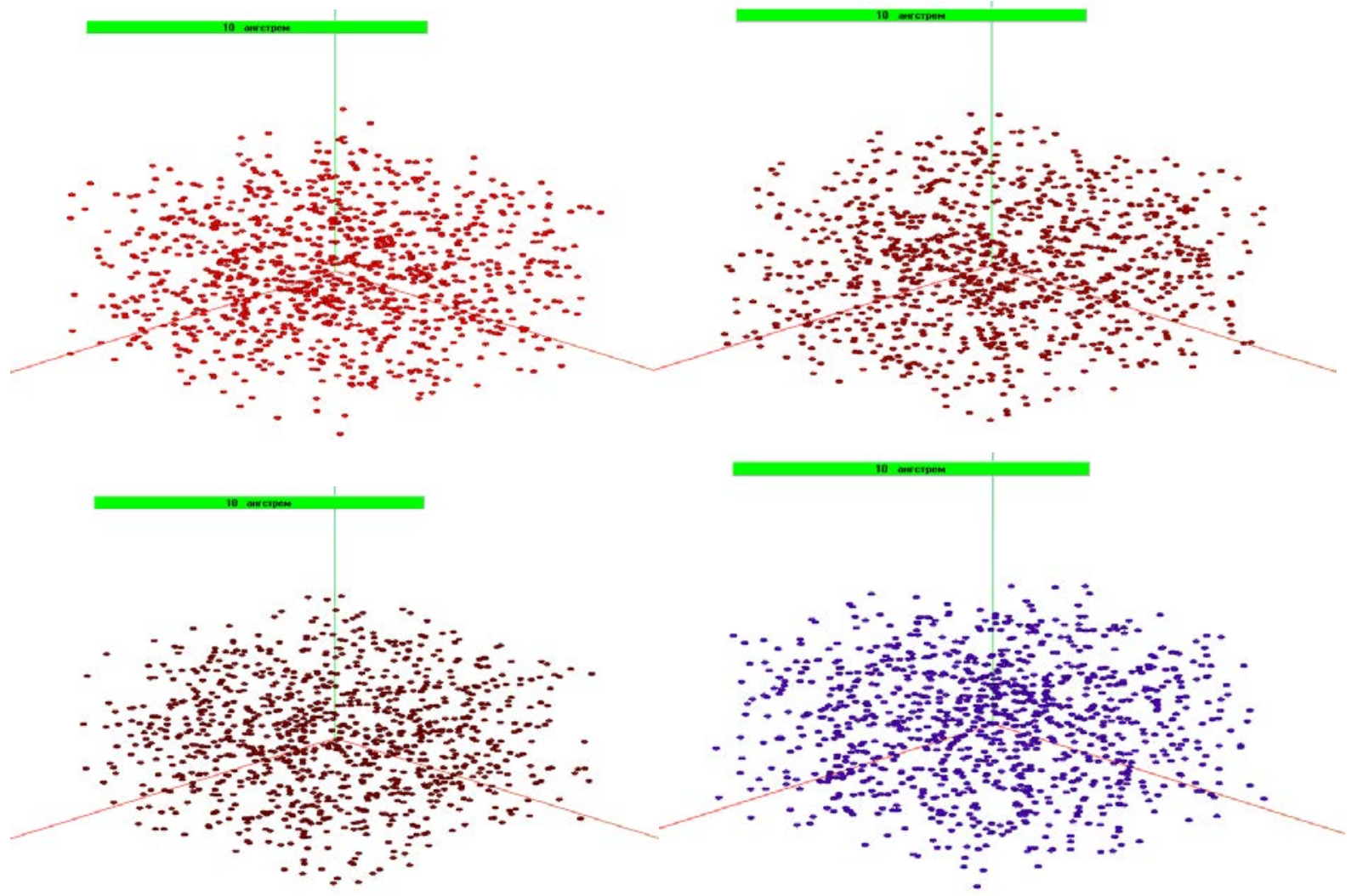

Рисунок 4 - Процесс охлаждения, с учетом граничных условий (1000 атомов).

Полученная модель может быть использованна при изучении различных процессов кристаллизации с условием дополнения ее алгоритмами образования отдельных доменных кристалических структур.

\section{Литература}

1. Кристаллические решетки. [Электронный ресурс]. URL: http://sernam.ru/lect_f_phis7.php?id=4 (дата обращения: 29.12.2013).

2. Теплофизические свойства стали. [Электронный ресурc]. URL: http://steelcast.ru/steel_properties (дата обращения: 29.12.2013).

3. Кристаллизация слитка спокойной стали. [Электронный ресурс]. URL: http://emchezgia.ru/razlivka/3_kristallizaciya_spokoynoy_stali.php (дата обращения: 29.12.2013).

4. ОБЩАЯ ХАРАКТЕРИСТИКА МЕТАЛЛОВ И СПЛАВОВ. [Электронный ресурс]. URL: http://www.naukaspb.ru/spravochniki/Demo\%20Metall/1.htm (дата обращения: 29.12.2013).

5. Атом. Материал из Википедии — свободной энциклопедии. [Электронный ресурс]. URL: http://ru.wikipedia.org/wiki/\%C0\%F2\%EЕ\%EC (дата обращения: 29.12.2013). 